\title{
The association between smoking and knee osteoarthritis in a cohort of Danish patients undergoing knee arthroscopy
}

\author{
Marianne Bakke Johnsen ${ }^{1,2^{*}}$, Kenneth Pihl ${ }^{3}$, Nis Nissen ${ }^{4}$, Rasmus Reinholdt Sørensen ${ }^{5}$, Uffe Jørgensen ${ }^{6}$,
} Martin Englund ${ }^{7}$ and Jonas Bloch Thorlund ${ }^{3}$

\begin{abstract}
Background: It has been suggested that smoking is associated with reduced risk of knee osteoarthritis (OA). However, supplementary studies are needed to further investigate any such potential association. Thus, our aim was to examine the relationship between smoking and early or more established knee OA in a cohort of relatively young patients with meniscal tears.

Methods: This cross-sectional study included 620 participants from the Knee Arthroscopy Cohort Southern Denmark (KACS) undergoing knee arthroscopy for a meniscal tear (mean age 49.2 (18.0-76.8) years). Recruitment of patients was performed between February 1, 2013, and January 31, 2015, at four different hospitals in Denmark. We defined early or more established knee $\mathrm{OA}$ as the combination of patient-reported frequent knee pain, degenerative meniscal tissue and presence of cartilage defects assessed by the operating surgeons. The relationship between smoking status and knee OA was examined by risk ratio (RR) with a 95\% confidence interval (CI), estimated from logistic regression adjusted for age, sex, BMI, education, work status and level of physical activity.

Results: The prevalence of early or more established knee OA was $37.7 \%$ in current smokers and $45.0 \%$ in non-smokers. We found no statistically significant association between current smoking and knee OA (adjusted RR 1.09, 95\% Cl 0.91-1.30).

Conclusions: This study found no relationship between current smoking and early or more established knee OA in a cohort of patients undergoing arthroscopic meniscal surgery. Thus, the inverse association between smoking and knee OA that has been suggested by previous studies was not confirmed.
\end{abstract}

Keywords: Smoking, Knee osteoarthritis, Meniscal tear, Knee arthroscopy, Risk factor

\section{Background}

An inverse association between smoking and knee osteoarthritis $(\mathrm{OA})$ has been suggested to exist, meaning that smoking may be protective of OA. A recent meta-analysis reported that this inverse relationship seems to exist regardless of study design, and that the association is stronger in men compared to women [1]. However, not all studies have identified this relationship between smoking and knee OA $[1,2]$. Currently, the exact

\footnotetext{
* Correspondence: m.b.johnsen@medisin.uio.no

${ }^{1}$ Institute of Clinical Medicine, University of Oslo, Oslo, Norway

${ }^{2}$ Research and Communication Unit for Musculoskeletal Health, Oslo

University Hospital, Building 37B, PO Box 4956, N-0424 Nydalen, Oslo,

Norway

Full list of author information is available at the end of the article
}

mechanisms by which smoking might protect against $\mathrm{OA}$ are not clear, although a positive effect of nicotine on chondrocyte metabolism has been proposed by in vitro studies $[3,4]$. On the contrary, studies have demostrated inconsistent results when studying the effect of smoking in patients by assessing articular cartilage volume using MRI [5, 6]. As any causal relationship between smoking and knee OA is still speculative, there is a need for studies to further elucidate this association. In previous studies, mainly radiographic definitions of knee OA or total knee replacement have been used to define the presence of severe knee OA [1]. Individuals with degenerative meniscal tears often exhibit early signs of knee $\mathrm{OA}$ and are considered to have a higher risk of

(c) The Author(s). 2019 Open Access This article is distributed under the terms of the Creative Commons Attribution 4.0 International License (http://creativecommons.org/licenses/by/4.0/), which permits unrestricted use, distribution, and reproduction in any medium, provided you give appropriate credit to the original author(s) and the source, provide a link to the Creative Commons license, and indicate if changes were made. The Creative Commons Public Domain Dedication waiver (http://creativecommons.org/publicdomain/zero/1.0/) applies to the data made available in this article, unless otherwise stated. 
developing the disease [7] and may therefore be a useful population to study the relationship between early degenerative knee changes and smoking. Therefore, the purpose of this study was to examine whether there is a relationship between smoking and early or more established knee OA in a cohort of patients with meniscal tears.

\section{Methods}

This cross-sectional study included participants from the Knee Arthroscopy Cohort Southern Denmark (KACS), which is a longitudinal cohort following patients undergoing knee arthroscopy for a meniscal tear [8]. The KACS cohort recruitment and inclusion criteria have previously been described in more detail in a protocol paper by Thorlund et al. [8], and by Pihl et al. [9]. In short, all patients were recruited between February 1, 2013 and January 31, 2015. Patients who were 18 years and older, and who were assigned for knee arthroscopy based on suspicion of a medial and/or lateral meniscus tear were included. In addition, patients had to understand and be able to read Danish and have an email address $[8,9]$. Further, exclusion criteria were: self-reported planned or previous anterior or posterior cruciate ligament (ACL or PCL) reconstruction in either knee, fracture(s) to either leg within the last six months at time of recruitment or not being able to answer the questionnaire due to mental impairment $[8,9]$. The Regional Scientific Ethics Committee of Southern Denmark exempted the study for ethical approval, as the study only utilizes questionnaire and register data. Still, all patients who participated in the study signed a written informed consent form [8].

\section{Exposure definition}

Main exposure was smoking status, and this data was collected through an email-based questionnaire prior to the arthroscopic surgery (median of 7 days prior to surgery, IQR 3-10 days), as reported previously [9]. Participants were asked: "Do you smoke?". The reply options were: "no", "no, stopped within the last six months", "yes, rarely", "yes, 1-5 cigarettes a day", "yes, 6-10 cigarettes a day", "yes, 11-20 cigarettes a day", "yes, more than 20 cigarettes a day", "yes, smoke pipe". Non-smokers were defined as those reporting "no" or "no, stopped within the last 6 months". Never and former smokers were both categorized as non-smokers $(n=482)$ due to the low number of former smokers $(n=$ 12). Current smokers were defined as those who reported smoking cigarettes (regardless of number of cigarettes) or a pipe. Surgeons were not aware of what the patients had replied in the email-based questionnaire.

\section{Covariates}

Relevant covariates that were available from the emailbased questionnaire were age, sex, highest completed level of education, work status, body mass index (BMI; calculated from baseline self-reported height and weight) and physical activity in leisure time assessed using a previously validated question $[10,11]$. Participants were asked "What education do you have?". The reply options were: "primary school", "high school", "vocational education", "short-cycle higher education", "medium higher education", "bachelor degree", "master", "PhD" and "other". We categorized education into primary school, high school/vocational training (including short-cycle higher education), bachelor level (including medium higher education) or master/PhD/other. Work status was assessed by asking "Are you currently working?", with reply options: "full time", "part time", "retired", "unemployed", "student" and "other". We categorized work status into employed (fulltime/part time/student/ other) or unemployed (retired/unemployed). Physical activity in leisure time was defined as low (hardly any activity, or light, non-strenuous activity once a week), medium (regular activity at least once a week) and high (regular, strenuous activity more than once a week).

\section{Knee symptoms}

Additionally, patients answered the Knee injury and Osteoarthritis Outcome Score (KOOS) prior to surgery. The KOOS questionnaire comprises 5 individual subscales: pain, symptoms, activities of daily living (ADL), sport and recreation function (Sport/Rec) and knee related quality of life (QoL), as described previously by Roos et al. [12]. The range of each subscale is from 0 to 100 points, with 0 indicating extreme knee problems and 100 indicating no knee problems. The KOOS has shown to be a valid, reliable and responsive patient-reported outcome measure for patients with several types of knee injuries and knee OA [13].

\section{Knee pathology at arthroscopy}

At the time of surgery, the operating surgeon recorded information about the quality of the meniscal tissue (i.e. non-degenerative, degenerative or undetermined) and degree of cartilage defects in each knee joint compartment. We classified cartilage defects as described previously in the KACS cohort [9], using the International Cartilage Repair Society (ICRS) grading system (grade 0 to 4) [14], where grade 0 represents normal cartilage and 4 represents severe cartilage lesions.

\section{Outcome definition}

We defined early or more established knee OA, as described by Pihl et al. [9], based on the combination of frequent knee pain, using only one item from the KOOS 
pain subscale (response alternatives "daily" or "always"), degenerative meniscal tissue (assessed by the surgeon at time of surgery) and cartilage defect (i.e. ICRS grade 1 in at least two knee joint compartments or at least ICRS grade 2 in one compartment). The outcome was categorized as a binary variable; early or more established knee $\mathrm{OA}$ versus no knee $\mathrm{OA}$.

\section{Statistical analysis}

Descriptive cohort characteristics are presented as means and standard deviations (SD) or numbers and percentages. We used a Chi squared test or an unpaired t-test, as appropriate, to compare descriptive characteristics. The relationship between smoking and knee OA was calculated by logistic regression with estimation of the risk ratio (RR) with a 95\% confidence interval (CI), using the method described by Norton et al. [15]. The full model was adjusted for age, sex, BMI, education, work status and level of physical activity. In a sensitivity analysis, we excluded adjustment for BMI due to the potential of collider bias [16]. We tested for interaction between smoking and sex based on previous results [1]. We compared the model with and without the interaction term using the likelihood ratio test. Finally, we checked the residuals and leverage of the model. We used Stata/SE 14.1 to perform all statistical analyses. $P$-value less than 0.05 was considered to be statistically significant.

\section{Results}

The study sample comprised 620 patients. Of these, $22.3 \%$ were current smokers and $77.7 \%$ were non-smokers. Current smokers compared with non-smokers were younger at age, had somewhat lower BMI and fewer had completed higher education (Table 1). Moreover, current smokers reported worse knee symptoms and function, according to the KOOS score, compared to non-smokers (Table 2). The prevalence of early or more established knee OA (as defined) was 37.7\% (52 of 138) in current smokers and $45.0 \%$ (217 of 482) in non-smokers. Of those with knee OA $(n=269), 37.2 \%$ had ICRS grade 1 in at least two knee joint compartments or ICRS grade 2 in one compartment, while $62.8 \%$ had ICRS grade 3 or 4 in at least one knee compartment. There was no statistically significant relationship between current smoking and knee $\mathrm{OA}$ in any of the models (Fig. 1). The results did not change substantially after excluding adjustment for BMI (RR 1.05, 95\% CI 0.88-1.27), nor did we find any interaction between smoking and sex ( $\mathrm{p}$ interaction term $=0.76$ ).

\section{Discussion}

In this study, we found no evidence of an association between current smoking and early or more established
Table 1 Patient characteristics

\begin{tabular}{|c|c|c|c|}
\hline Variables & $\begin{array}{l}\text { Current smokers } \\
(n=138)\end{array}$ & $\begin{array}{l}\text { Non-smokers } \\
(n=482)\end{array}$ & $P$-value \\
\hline Age, years (SD) & $45.0(12.2)$ & $50.4(12.9)$ & $<0.001$ \\
\hline $\mathrm{BMI}, \mathrm{kg} / \mathrm{m}^{2}$ (SD) & $26.7(4.4)$ & $27.4(4.4)$ & 0.08 \\
\hline Female sex, n (\%) & $54(39.1)$ & $213(44.2)$ & 0.29 \\
\hline \multicolumn{4}{|l|}{ Education, n (\%) } \\
\hline Primary school & $28(20.3)$ & $73(15.2)$ & \\
\hline $\begin{array}{l}\text { High school/vocational } \\
\text { training }\end{array}$ & $68(49.3)$ & $247(51.2)$ & \\
\hline Bachelor level & $40(29.0)$ & $119(24.7)$ & \\
\hline Master/PhD/other & $2(1.5)$ & $43(8.9)$ & 0.01 \\
\hline \multicolumn{4}{|l|}{ Work status, n(\%) } \\
\hline Unemployed & $27(19.6)$ & $119(24.7)$ & \\
\hline $\begin{array}{l}\text { Employed (or self- } \\
\text { employed) }\end{array}$ & $111(80.4)$ & $363(75.3)$ & 0.21 \\
\hline \multicolumn{4}{|l|}{ Physical activity, n (\%) } \\
\hline Low & $35(25.3)$ & $92(19.1)$ & \\
\hline Medium & $51(37.0)$ & $190(39.4)$ & \\
\hline High & $52(37.7)$ & $200(41.5)$ & 0.27 \\
\hline
\end{tabular}

$B M I$ body mass index

${ }^{a}$ Chi squared test for categorical variables and an unpaired t-test for continuous variables

knee OA. Thus, the potential inverse relationship between smoking and knee OA could not be confirmed.

In contrast to the meta-analysis that reported an inverse relationship between smoking and knee OA to be independent of study design [1], a previous study including individuals from the Osteoarthritis Initiative (OAI), with knee OA based on Kellgren-Lawrence (KL) grade $\geq 2$, found an association between smoking and knee symptoms and structural disease status that was dependent on study design [2]. The cross-sectional analysis indicated worse knee function but greater joint space width (JSW) in current smokers compared to never smokers. However, there was no difference in symptom changes or JSW between smokers and never smokers in the longitudinal analysis. The authors believed the longitudinal analysis to be more robust and concluded that the differences found in the cross-sectional analysis could be due to residual confounding [2]. In the Clearwater Osteoarthritis Study, a prospective study of 2505 individuals, there was an indication of a protective effect of current smoking on the risk of OA in the hand, hip, knee and foot in unadjusted analyses [17]. However, after the adjustment of covariates such as age, gender, BMI and physical activity [17] the relationship disappeared; this was also observed in the present study.

Similar to participants in the Osteoarthritis Initiative [2], smokers in the current study reported worse knee symptoms and function compared to non-smokers 
Table 2 Description of patient symptoms and knee pathology

\begin{tabular}{llll}
\hline Variables & $\begin{array}{l}\text { Current smokers } \\
(n=138)\end{array}$ & $\begin{array}{l}\text { Non-smokers } \\
(n=482)\end{array}$ & $P_{\text {-value }}{ }^{\mathrm{d}}$ \\
\hline Frequency of knee pain ${ }^{\mathrm{a}}, \mathrm{n}(\%)$ & & \\
Never & $3(2.2)$ & $6(1.2)$ & \\
Monthly & $7(5.1)$ & $21(4.4)$ & \\
Weekly & $15(10.9)$ & $55(11.4)$ & \\
Daily & $88(63.8)$ & $313(64.9)$ & \\
Always & $25(18.1)$ & $87(18.1)$ & 0.94 \\
KOOS scores ${ }^{b}$, mean $(95 \%$ Cl) & & \\
Pain & $52.3(49.2-55.3)$ & $55.6(54.0-57.3)$ & 0.06 \\
Symptoms & $56.6(53.5-59.7)$ & $60.9(59.2-62.5)$ & 0.02 \\
ADL & $60.0(56.8-63.1)$ & $64.8(63.0-66.5)$ & 0.01 \\
Sport/Rec & $22.5(19.2-25.9)$ & $27.3(25.3-29.3)$ & 0.02 \\
QoL & $37.9(35.3-40.5)$ & $42.7(41.3-44.0)$ & 0.001
\end{tabular}

Meniscal tissue quality, $\mathrm{n}(\%)$

\begin{tabular}{|c|c|c|c|}
\hline Non-degenerative & $65(47.1)$ & $180(37.3)$ & \\
\hline Degenerative & $69(50.0)$ & $286(59.3)$ & \\
\hline Undetermined & $4(2.9)$ & $16(3.3)$ & 0.11 \\
\hline \multicolumn{4}{|c|}{ ICRS cartilage grade ${ }^{c}, \mathrm{n}(\%)$} \\
\hline \multicolumn{4}{|c|}{ Medial compartment } \\
\hline Grade 0 & $42(30.4)$ & $136(28.2)$ & \\
\hline Grade 1 & $38(27.5)$ & $108(22.4)$ & \\
\hline Grade 2 & $28(20.3)$ & $88(18.3)$ & \\
\hline Grade 3 & $28(20.3)$ & $111(23.0)$ & \\
\hline Grade 4 & $2(1.5)$ & $39(8.1)$ & 0.06 \\
\hline \multicolumn{4}{|c|}{ Lateral compartment } \\
\hline Grade 0 & $66(47.8)$ & $197(40.9)$ & \\
\hline Grade 1 & $48(34.8)$ & $158(32.8)$ & \\
\hline Grade 2 & $15(10.9)$ & $77(16.0)$ & \\
\hline Grade 3 & $9(6.5)$ & $36(7.5)$ & \\
\hline Grade 4 & 0 & $14(2.9)$ & 0.11 \\
\hline \multicolumn{4}{|c|}{ Patellofemoral compartment } \\
\hline Grade 0 & $54(39.1)$ & $174(36.1)$ & \\
\hline Grade 1 & $45(32.6)$ & $122(25.3)$ & \\
\hline Grade 2 & $19(13.8)$ & $85(17.6)$ & \\
\hline Grade 3 & $16(11.6)$ & $73(15.2)$ & \\
\hline Grade 4 & $4(2.9)$ & $28(5.8)$ & 0.20 \\
\hline
\end{tabular}

${ }^{\mathrm{a}}$ Single item from the KOOS pain subscale

${ }^{\mathrm{b}} \mathrm{Score}$ ranging from 0 to 100 , with 0 indicating extreme problems and 100 indicating no problems

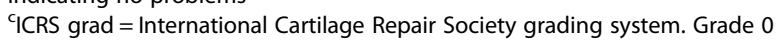
representing normal cartilage and 4 representing very severe cartilage lesion ${ }^{d}$ Chi squared test for categorical variables and an unpaired $t$-test for continuous variables

$A D L$ activities of daily living, Sport/Rec sport and recreational activities, QoL quality of life

according to the subscales of KOOS. However, the difference was small and probably not clinically important as differences around 10 points are usually considered to constitute a minimal clinically relevant difference in KOOS subscales [13]. We observed no statistically significant difference between smokers and non-smokers in self-reported knee pain. Smoking has been suggested to have an analgesic effect through the constituent of nicotine [18]. Nevertheless, smokers have also been found to have a higher risk of musculoskeletal pain compared to non-smokers [18]. Smokers also tend to report more intense pain than non-smokers when musculoskeletal pain is present [18]. In the current study, however, all participants were expected to have a certain degree of knee pain, regardless of smoking status, as all patients underwent surgery for a meniscal tear and knee pain.

One hypothesis is that smoking might decrease the risk of knee OA through a positive effect of nicotine on articular chondrocyte function [4, 6]. In a sample of 297 adults without knee OA or any history of knee injury from the Melbourne Collaborative Cohort Study, ever smoking was associated with an increase in tibial cartilage volume, when compared to never smoking, but not with the presence of cartilage defects [6]. Furthermore, the study reported a positive association, suggesting a dose-dependent relationship between the number of pack-years and cartilage volume [6]. We did not assess cartilage volume in our study. However, the operating surgeons in our study reported a greater proportion of degenerative meniscal tissue quality in non-smokers versus current smokers (59.3\% vs $50.0 \%$ ), though this was not statistically significant. Our crude analysis supported this descriptive finding, indicating a reduced prevalence of knee OA among current smokers compared to non-smokers. However, after adjustments, mainly for age, the direction of the association changed. A second hypothesis is that smoking might affect the risk of knee OA through its effect on BMI [16]. However, one former study showed that the indirect effect of smoking on hip and knee OA was small [19], as was indicated by the sensitivity analysis in the present study.

This study has important limitations. For instance, its cross-sectional design limits the ability to make causal inferences. We used self-reported smoking status as the main exposure, which is a crude measure of smoking exposure with risk of misclassification. Further, we categorized former smokers who had stopped smoking within the last 6 months as "non-smokers". However, the number of former smokers was low $(n=12)$. A misclassification may have decreased any potential differences between current and non-smokers because of similarities in the smoking exposure of the two groups. Although surgeons were blinded to the patients' responses to smoking status in the email-based questionnaire, patients may have made the surgeon aware of it during the consultation. 


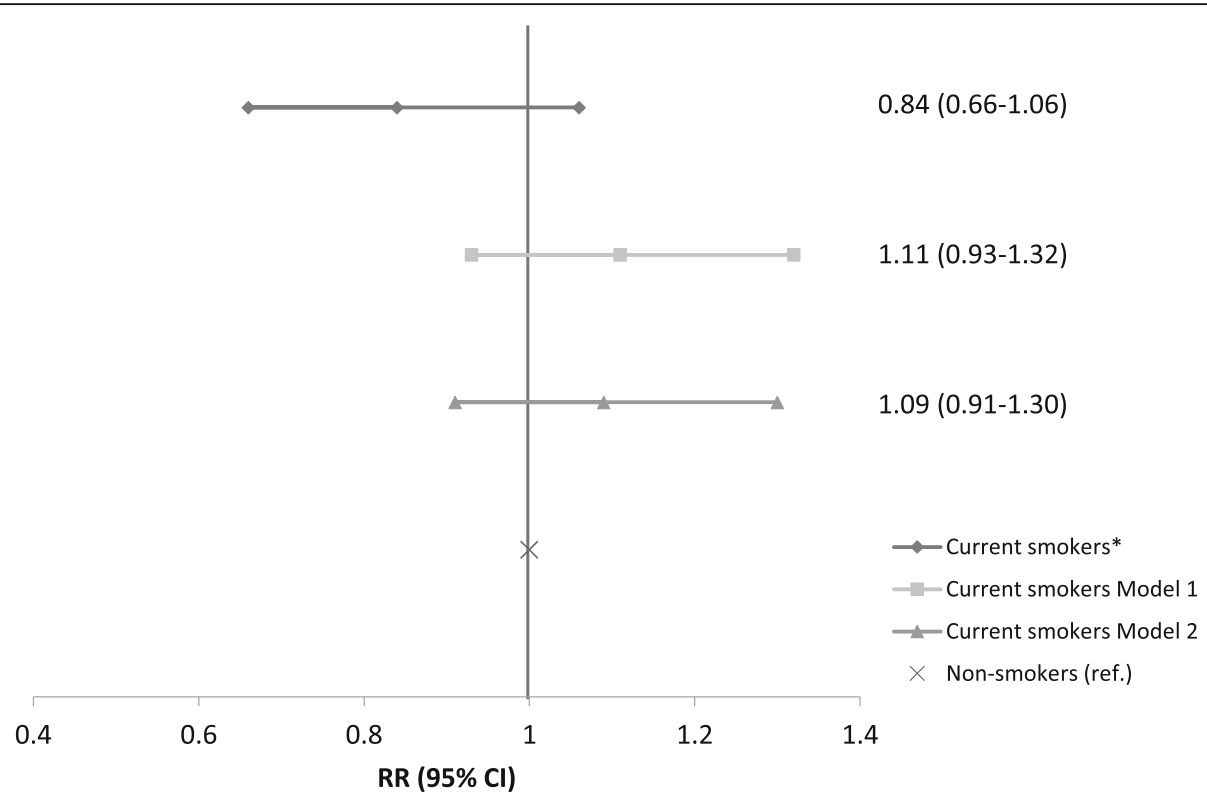

Fig. 1 Association between smoking and early or more established knee OA. *Crude: logistic regression with estimation of risk ratio (RR) and 95\% Cl. Model 1: logistic regression with RR and 95\% Cl, adjusted for age, sex and BMI. Model 2: logistic regression with RR and 95\% Cl, adjusted for age, sex, BMl, highest completed level of education, work status and the level of leisure time physical activity

However, at the time of surgery, the surgeons were not aware of any particular intention to study the association between smoking and cartilage status. Thus, it is unlikely that knowledge of the patient's smoking status would have influenced their cartilage grading. Due to the small sample size we did not further stratify the main analysis, e.g. by OA severity, gender or age. However, as mentioned, there was no sign of statistical interaction between smoking and sex. We only investigated subjects selected for knee arthroscopy and it is unclear whether smoking status or other patient characteristics may have influenced this selection for surgery, thereby affecting our results. In addition, since all patients had a certain degree of knee pain and cartilage degeneration, we cannot disregard that this might have attenuated the potential association between smoking and knee OA. However, the proportion of smokers in this cohort (22\%) is similar to the general Danish population [20]. To the best of our knowledge, this is the first study to investigate the association between smoking and early or more established knee OA, as currently defined, in a relatively young cohort of patients. Previous studies have primarily used $\mathrm{KL} \geq 2$ or total knee replacement as a measure of radiographic or severe knee OA and patients being substantially older. It has been shown that meniscal tears are associated with $\mathrm{OA}$ even in knees with $\mathrm{KL}$ grade 0 , thus, degenerative meniscal tears are thought to be one of the key factors in the early development of knee OA [7].

\section{Conclusion}

In conclusion, this study found no relationship between current smoking and early or more established knee OA in a cohort of patients undergoing knee arthroscopy for a meniscal tear. Meniscal tears, cartilage defects and knee pain may be important markers of knee OA and represent a different phenotype of knee OA than has previously been studied in relation to smoking.

\section{Abbreviations}

ADL: Activities of daily living; BMI: Body mass index; Cl: Confidence intervals; ICRS: International Cartilage Repair Society; IQR: Interquartile range; JSW: Joint space width; KACS: Knee Arthroscopy Cohort of Southern Denmark; KL: Kellgren-Lawrence; KOOS: Knee injury and Osteoarthritis Outcome Score; MRI: Magnetic resonance imaging; OA: Osteoarthritis; QoL: Quality of life; RR: Risk ratio; SD: Standard deviations

\section{Acknowledgements}

The authors would like to thank all participating patients as well as the orthopedic surgeons, nurses and secretaries at the Department of Orthopaedic Surgery and Traumatology, Odense University Hospital (Odense and Svendborg) and the Department of Orthopaedic Surgery, Lillebaelt Hospital (Kolding and Vejle) for their assistance in patient recruitment and data collection.

\section{Funding}

The KACS cohort was funded by an individual postdoctoral grant (JBT) from the Danish Council for Independent Research/Medical Sciences and funds from the Region of Southern Denmark. MBJ was supported by an individual postdoctoral grant from the Research Council of Norway (grant number 248817). The funding sources had no involvement in any aspect of this manuscript.

\section{Availability of data and materials}

The data supporting the findings of this study are available from the last author (JBT, jthorlund@health.sdu.dk) upon reasonable request. 


\section{Authors' contributions}

MBJ, JBT and ME contributed to the conception and design of the study. $\mathrm{NN}, \mathrm{UJ}$ and RRS participated in setting up the study, patient recruitment and data collection. MBJ and KP performed the analysis of the data, while all authors contributed to the interpretation of data. MBJ and JBT drafted the first version of the manuscript. All authors contributed to critical revision of the manuscript, and read and approved the final version.

\section{Ethics approval and consent to participate}

The study was conducted in accordance with the Declaration of Helsinki. All patients provided written informed consent to participate in the study. The Regional Scientific Ethics Committee of Southern Denmark waived the need for ethical approval as the study only involved questionnaire and register data.

\section{Consent for publication}

Not applicable.

\section{Competing interests}

JBT is an Associate Editor for BMC Musculoskeletal Disorders.

\section{Publisher's Note}

Springer Nature remains neutral with regard to jurisdictional claims in published maps and institutional affiliations.

\section{Author details}

${ }^{1}$ Institute of Clinical Medicine, University of Oslo, Oslo, Norway. ${ }^{2}$ Research and Communication Unit for Musculoskeletal Health, Oslo University Hospital, Building 37B, PO Box 4956, N-0424 Nydalen, Oslo, Norway. ${ }^{3}$ Research Unit for Musculoskeletal Function and Physiotherapy, Department of Sports Science and Clinical Biomechanics, University of Southern Denmark, Odense, Denmark. ${ }^{4}$ Lillebaelt Hospital, Kolding, Denmark. ${ }^{5}$ Department of Orthopaedic Surgery, Lillebaelt Hospital, Vejle, Denmark. ${ }^{6}$ Odense University Hospital, Odense, Denmark. ${ }^{7}$ Clinical Epidemiology Unit, Orthopedics, Department of Clinical Sciences Lund, Faculty of Medicine, Lund University, Lund, Sweden.

Received: 4 June 2018 Accepted: 19 March 2019

Published online: 01 April 2019

\section{References}

1. Kong L, Wang L, Meng F, Cao J, Shen Y. Association between smoking and risk of knee osteoarthritis: a systematic review and meta-analysis. Osteoarthr Cartil. 2017:25:809-16.

2. Dube CE, Liu SH, Driban JB, MCAlindon TE, Eaton CB, Lapane KL. The relationship between smoking and knee osteoarthritis in the osteoarthritis initiative. Osteoarthr Cartil. 2016;24:465-72.

3. Gullahorn L, Lippiello L, Karpman R. Smoking and osteoarthritis: differential effect of nicotine on human chondrocyte glycosaminoglycan and collagen synthesis. Osteoarthr Cartil. 2005;13:942-3.

4. Ying X, Cheng S, Shen Y, Cheng X, An Rompis F, Wang W, et al. Nicotine promotes proliferation and collagen synthesis of chondrocytes isolated from normal human and osteoarthritis patients. Mol Cell Biochem. 2012;359:263-9.

5. Davies-Tuck ML, Wluka AE, Forbes A, Wang Y, English DR, Giles GG, et al. Smoking is associated with increased cartilage loss and persistence of bone marrow lesions over 2 years in community-based individuals. Rheumatology (Oxford). 2009;48:1227-31.

6. Racunica TL, Szramka M, Wluka AE, Wang Y, English DR, Giles GG, et al. A positive association of smoking and articular knee joint cartilage in healthy people. Osteoarthr Cartil. 2007;15:587-90.

7. Englund M, Guermazi A, Roemer FW, Aliabadi P, Yang M, Lewis CE, et al. Meniscal tear in knees without surgery and the development of radiographic osteoarthritis among middle-aged and elderly persons: the multicenter osteoarthritis study. Arthritis Rheum. 2009;60:831-9.

8. Thorlund JB, Christensen R, Nissen N, Jorgensen U, Schjerning J, Porneki JC, et al. Knee arthroscopy cohort Southern Denmark (KACS): protocol for a prospective cohort study. BMJ Open. 2013;3:e003399.

9. Pihl K, Englund M, Lohmander LS, Jorgensen U, Nissen N, Schjerning J, et al. Signs of knee osteoarthritis common in 620 patients undergoing arthroscopic surgery for meniscal tear. Acta Orthop. 2017;88:90-95.
10. Johansson G, Westerterp KR. Assessment of the physical activity level with two question: validation with doubly labeled water. Int J Obes. 2008;32: 1031-3.

11. Saltin B, Grimby G. Physiological analysis of middle-aged and older former athletes. Comparison with still active athletes of the same age. Circulation. 1968;38:1104-15.

12. Roos EM, Roos HP, Lohmander LS, Ekdahl C, Beynnon BD. Knee injury and osteoarthritis outcome score (KOOS)--development of a self-administered outcome measure. J Orthop Sports Phys Ther. 1998;28:88-96.

13. Roos EM, Lohmander LS. The knee injury and osteoarthritis outcome score (KOOS): from joint injury to osteoarthritis. Health Qual Life Outcomes. 2003;1:64

14. Brittberg $M$, Winalski CS. Evaluation of cartilage injuries and repair. J Bone Joint Surg Am. 2003;85-A(Suppl 2):58-69.

15. Norton EC, Miller MM, Kleinman LC. Computing adjusted risk ratios and risk differences in Stata. Stata J. 2013;13:492-509.

16. Felson DT, Zhang Y. Smoking and osteoarthritis: a review of the evidence and its implications. Osteoarthr Cartil. 2015;23:331-3.

17. Wilder FV, Hall BJ, Barrett JP. Smoking and osteoarthritis: is there an association? The Clearwater osteoarthritis study. Osteoarthr Cartil. 2003;11:29-35.

18. Shi Y, Weingarten TN, Mantilla CB, Hooten WM, Warner DO. Smoking and pain: pathophysiology and clinical implications. Anesthesiology. 2010;113: 977-92.

19. Johnsen MB, Hellevik Al, Smastuen MC, Langhammer A, Furnes O, Flugsrud $\mathrm{GB}$, et al. The mediating effect of body mass index on the relationship between smoking and hip or knee replacement due to primary osteoarthritis. A population-based cohort study (the HUNT study). PLoS One. 2017;12:e0190288

20. Hoffman SH, Schramm S, Jarlstrup NS, Christensen Al. Danskenes rygevaner. Udviklingen fra 1994 til 2017. Sundhedsstyrelsen. Statens Institut for Folkesundhed, SDU. (Accessed 17 Jan 19). Available from: https://www.sst. dk/da/udgivelser/2019/danskernes-rygevaner-udviklingen-fra-1994-2017

\section{Ready to submit your research? Choose BMC and benefit from:}

- fast, convenient online submission

- thorough peer review by experienced researchers in your field

- rapid publication on acceptance

- support for research data, including large and complex data types

- gold Open Access which fosters wider collaboration and increased citations

- maximum visibility for your research: over $100 \mathrm{M}$ website views per year

At BMC, research is always in progress.

Learn more biomedcentral.com/submissions 